\title{
Las revistas científicas en América Latina hacia el camino del acceso abierto: un diagnóstico de políticas y estrategias editoriales
}

\author{
Scientific journals in Latin America on their way \\ towards open access: A diagnosis on policies \\ and editorial strategies
}

\author{
Nancy SÁNCHEZ-TARRAGÓ' \\ Alejandro CABALLERO-RIVERO² \\ Piotr TRZESNIAK ${ }^{3}$ \\ Dania DEROY DOMÍNGUEZ ${ }^{4}$ \\ Raimundo Nonato Macedo dos SANTOS ${ }^{3}$ \\ Juan-Carlos FERNÁNDEZ-MOLINA ${ }^{5}$
}

\section{Resumen}

Este trabajo presenta los resultados más relevantes de un diagnóstico sobre el estado de las políticas y estrategias de los comités editoriales de las revistas científicas latinoamericanas para favorecer el uso y reúso en acceso abierto de la producción científica que publican, así como sobre las percepciones y actitudes de editores y autores con relación a estas políticas y estrategias. Se realizó análisis cuantitativo y cualitativo a los datos obtenidos de 516 revistas y de 400 cuestionarios de editores y 101 de autores. La mayoría de las revistas son gratuitas y consideradas de acceso abierto por sus editores, pero en muchos casos disponen de políticas de derecho de autor restrictivas o carecen de información explícita sobre las condiciones de uso de los artículos. Las estrategias institucionales y editoriales para promover el modelo son escasas y tienen poca visibilidad entre los autores. El financiamiento sostenible y la falta de familiarización con el modelo de acceso abierto están entre las barreras más importantes para que América Latina lleve adelante estas iniciativas. La región de América Latina necesita mayor y mejor alineación y coherencia de las políticas institucionales y editoriales con los principios y estrategias que favorecen el acceso abierto, así como mayor preparación de editores y autores.

Palabras clave: Acceso abierto. América Latina. Derecho de autor. Políticas editoriales. Revistas científicas.

\footnotetext{
Abstract

This paper presents the main results of a study carried out in order to assess the current state of policies and strategies used by editorial boards of Latin American scientificjournals, to promote use and reuse of open access scientific production, as well as to identify publishers

1 Universidade Federal de Pernambuco, Centro de Artes e Comunicação, Departamento de Ciência da Informação. Av. Prof. Moraes Rego, 1235, Cidade Universitária, 50740-550, Recife, PE, Brasil. Correspondencia a nombre de/Correspondence to: N. SÁNCHEZ-TARRAGÓ. E-mail: <nancita1973@gmail.com>.

2 Academia de Ciencias de Cuba. Habana Vieja, Cuba.

3 Universidade Federal de Pernambuco, Centro de Artes e Comunicação, Programa de Pós-Graduação em Ciências da Informação. Recife, PE, Brasil.

${ }^{4}$ Facultad Latinoamericana de Ciencias Sociales, Biblioteca Marina Majoli. La Habana, Cuba.

5 Universidad de Granada, Facultad de Comunicación y Documentación, Colegio Máximo de Cartuja. Granada, España.

Recebido el 3/3/2015, re-presentado el 29/6/2015 y aceptado para su publicación el 4/8/2015.
} 
and authors' perceptions and attitudes regarding these policies and strategies. Quantitative and qualitative analysis was carried out on data obtained from 516 journals and questionnaires applied to 400 publishers and 101 authors. Although most of the journals are free and considered as open access by their publishers, actually, in many cases they have established restrictive copyright policies or lack explicit information on terms and conditions of use and publishing of their articles. Institutional and editorial strategies to promote open access are scarce and have little visibility among authors. Sustainable funding and lack of familiarity with open access model are among the most important barriers for Latin America push these initiatives forward. Latin America needs a better and larger alignment and coherence of institutional and editorial policies with principles and strategies that promote open access, as well as further training for editors and authors.

Keywords: Open access. Latin America. Copyright. Editorial policies. Scientific journals.

\section{Introducción}

Durante los últimos 20 años, la publicación científica ha estado en el centro de grandes transformaciones y cambios potenciados por la revolución digital, Internet y el World Wide Web. Dos etapas se manifiestan con mayor agudeza: la primera y más evidente, la evolución de las revistas impresas a revistas electrónicas o la coexistencia de versiones en ambos formatos. La segunda, el Movimiento de a Acceso Abierto, que promueve el acceso a los artículos científicos sin barreras económicas, tecnológicas o legales, según define la Declaración de Budapest (Budapest Open Access Initiative, 2002).

Acceso abierto significa que la literatura científica esté libremente accesible bajo condiciones generosas que permitan la mayor parte de las reutilizaciones, siempre y cuando el autor reciba el reconocimiento por su trabajo cuando éste se vuelva a publicar, sea adaptado o integrado a otros trabajos. Los usuarios de estas obras deberían tener derecho a una amplia gama de actividades creativas tales como traducción, combinación, análisis, adaptación y preservación (Carroll, 2013). Por ello, las transformaciones del modelo de comunicación científica que propugna el Movimiento de Acceso Abierto dependen también de cambios en aspectos esenciales dentro de las políticas científicas e institucionales, incluyendo las políticas de derecho de autor.

Aunque las principales declaraciones sobre el acceso abierto se gestaron en el hemisferio norte, América Latina ha sido una región con una participación importante en iniciativas encaminadas a proporcionar un amplio acceso al conocimiento. Un dato que corrobora esta afirmación es la fundación ya en 1998 de la Biblioteca Electrónica Scientific Electronic Library Online (SciELO, Biblioteca Científica Electrónica en Línea) <http:// www.scielo.org>, importante iniciativa para disponer en formato electrónico y en línea la ciencia latinoamericana publicada en revistas científicas nacionales. Otros ejemplos son la Red de Revistas Científicas de América Latina y el Caribe, España y Portugal (Redalyc) 2002, <http://www.redalyc.org/> y, más recientemente, Scientific Periodicals Electronic Library (SPELL) 2012, <http:// www. spell.org.br>. Babini (2011) expone el avance de servicios regionales de acceso abierto que ofrecen acceso libre al texto completo de publicaciones científicas y académicas de la región, sin costo para el usuario final y sin necesidad de suscripción. Análisis más particulares del acceso abierto en países latinoamericanos son expuestos en Gómez et al. (2009); Sánchez-Tarragó et al. (2012a) y Miguel et al. (2013).

No obstante estos avances, el estudio de Cerda Silva y Lara (2011) - único antecedente encontrado sobre el tema en América Latina que analizó los aspectos legales y tecnológicos presentes en las políticas editoriales de una muestra de revistas académicas latinoamericanas electrónicas -, evidenció que muchas revistas mantenían políticas de derecho de autor restrictivas así como inconsistencias entre los aspectos tecnológicos, que en muchos casos favorecían el acceso abierto, y los legales, que lo frenaban.

El trabajo que se presenta a continuación constituye parte de una investigación más amplia (Sánchez-Tarragó et al., 2013) que tuvo como objetivo profundizar en el estado de las políticas y estrategias institucionales y de los comités editoriales de las revistas científicas latinoamericanas para favorecer el uso y reúso en acceso abierto de la producción científica que publican, así como identificar las percepciones y actitudes de editores y autores acerca de estas políticas, estrategias y posibles barreras que obstaculizan la adopción del acceso abierto. Este artículo se propone, por tanto, presentar los hallazgos más relevantes del 
diagnóstico realizado sobre las políticas y estrategias de los comités editoriales, confrontando los resultados de los investigadores con las perspectivas de editores y autores. A partir de esta triangulación metodológica se ofrecen recomendaciones para los comités editoriales, las instituciones y otros actores del sistema de comunicación científica.

\section{Acceso abierto, derecho de autor y políticas y estrategias editoriales}

En el contexto del Movimiento de Acceso Abierto, las barreras legales o de permisos se refieren a las que impone el derecho de autor o copyright. Son aquellas que implican que los usuarios necesitan el permiso del titular del derecho de autor (en el ámbito de las revistas académicas, tradicionalmente es la editorial o revista) para usar y reusar los contenidos publicados. Suber (2012) argumenta que, como resultado de estas barreras, no solo se imponen obstáculos a los usuarios, sino que los autores se perjudican por los usos limitados que pueden darse a sus trabajos. A lo largo de los últimos años, y como resultado del empuje del Movimiento de Acceso Abierto, se han producido muchos cambios en los modelos de negocios de las editoriales y en el uso de los tradicionales acuerdos de transferencia de derechos de autor exigidos por estas (Villarroya et al., 2012; Ware \& Mabe, 2012). Cada vez más editoriales y revistas utilizan ya sea modelos de acceso abierto puros (ni los autores ni los usuarios pagan por los contenidos accesibles en acceso abierto) o modelos híbridos (donde el autor o su institución pagan una tarifa por hacer disponible en acceso abierto el contenido publicado). También, más editoriales y revistas utilizan licencias abiertas donde el autor retiene derechos que puede ceder al usuario de los contenidos, aunque todavía muchas revistas de acceso abierto no aprovechan al máximo las oportunidades que ofrecen estas licencias (Budapest Open Access Initiative, 2012). Las más conocidas, aunque no las únicas, son las licencias Creative Commons <https://creativecommons.org/>.

Como plantea Reinsfelder (2012), la complejidad de interrelaciones que se establece entre los diferentes actores del sistema de comunicación científica implica que cualquier discusión sobre el éxito del acceso abierto debe incluir a todos los involucrados (autores, editores, bibliotecarios, entre otros). Al respecto Pinch y Bijker (1987) señalan que durante la'construcción social de las tecnologías'los grupos sociales relevantes pueden tener diferentes interpretaciones de los significados que emanan de estas. A tenor de esto puede mencionarse que no obstante las transformaciones antes mencionadas sobre modelos de negocios y acuerdos de derecho de autor, según el informe de Ware y Mabe (2012) lo notorio del debate es que tiene lugar en medio de la ignorancia y los malentendidos acerca de su esencia y relevancia en el contexto actual.

Al respecto, el trabajo de Morris (2009) encontró que los autores subestimaban lo que ellos podían hacer con los preprint (por ejemplo, depositarlos en repositorios, usarlos en paquetes de cursos, enviarlos a colegas), mientras que sobrestimaban lo que las políticas editoriales les permitían hacer con las versiones publicadas. El estudio llega a la conclusión de que los editores no han comunicado sus políticas de derecho de autor con efectividad. Por otra parte, la confusión entre las licencias abiertas y los tradicionales acuerdos de derecho de autor (copyright) constituye un fenómeno que afecta actualmente a muchísimas revistas de acceso abierto en todas las latitudes. Un reciente artículo del blog que mantiene el conocido Directory of Open Access Journals (DOAJ) ejemplifica los mensajes confusos que se envían a autores y usuarios cuando en los sitios Web de las revistas aparecen declaraciones donde se mezcla el símbolo de copyright con la licencia abierta: @ [Publisher Name]. This work is licensed under a Creative Commons License and all rights remain with the author. Si además de eso - como añaden los autores del blog - los editores colocan el enlace de su declaración de derecho de autor (copyright) cuando DOAJ solicita el URL de la licencia abierta, también se puede pensar que no se trata de que los editores intenten deliberadamente confundir a los autores y usuarios, sino que ellos mismos están confundidos (Directory of Open Access Journals, 2015). En este contexto, por tanto, la preparación de los comités editoriales es fundamental, así como la adopción consciente de políticas y estrategias que favorezcan el acceso abierto.

Por otra parte, los estudios de Björk (2004), Swan y Brown (2005), Harnad (2006) y Xia (2010) identificaron que, a pesar de las percepciones y actitudes generalmente 
positivas de los autores hacia el acceso abierto, su participación real estaba influenciada por complejos factores sociológicos, sicológicos, económicos y tecnológicos. Particularmente, varios estudios Ilaman la atención sobre cómo la ausencia de un conocimiento adecuado sobre el derecho de autor y las políticas relacionadas con este, se ha convertido en una de las principales razones para la reticencia de los autores a depositar en los repositorios e, inclusive, a tener percepciones más favorables hacia las revistas de acceso abierto (Swan\&Brown, 2005; Fryetal.,2009; Dallmeier-Tiessen et al., 2011). En el ámbito de los países latinoamericanos, los primeros estudios que exploraron los conocimientos, actitudes y prácticas de los investigadores con relación al acceso abierto detectados en la revisión bibliográfica fueron los de Sánchez-Tarragó y Fernández-Molina (2008, 2010), en investigadores cubanos y Gómez et al. (2008), en chilenos. Posteriormente, otros trabajos han abordado estudios similares en comunidades académicas de Argentina (Bongiovani et al., 2012; Miguel et al., 2013). En sentido general, la falta de familiarización con los conceptos claves del Movimiento de Acceso Abierto y con los aspectos relativos al derecho de autor tiene influencia negativa en las prácticas relacionadas con el acceso abierto. Por tanto, pudiéramos afirmar que los conocimientos, percepciones y actitudes de cada uno de los actores del sistema pueden influir notablemente en acciones favorables (estrategias, iniciativas, prácticas) para la adopción del modelo de acceso abierto, o pueden constituir barreras.

De acuerdo con el análisis de Reinsfelsder (2012) sobre las bases de poder de los diferentes actores del sistema de comunicación científica, las editoriales y revistas ejercen una gran influencia sobre otros actores, como los autores y los profesionales de la información. Por ejemplo, pueden imponer licencias de derecho de autor restrictivas, obligar a que las ideas y datos se presenten de determinada forma, presionar a los bibliotecarios a firmar acuerdos para el acceso a sus contenidos, entre otros. Por otra parte, su influencia también les permite ofrecer a los autores vías para lograr una amplia visibilidad de sus ideas y proporcionar incentivos a los profesionales de la información a través de mejores precios, productos y servicios de valor añadido. A partir de estas consideraciones es posible sugerir que las políticas y estrategias que las editoriales y revistas adopten a favor del acceso abierto van a tener una influencia directa en el resto de los actores del sistema de comunicación científica.

Tal como plantean Cerda Silva y Lara, disponer de adecuadas políticas editoriales contribuye a que los contenidos publicados tengan una amplia distribución, acceso y uso. Pero no se trata simplemente de determinar la línea editorial o cuáles artículos se incluirán en un número "sino que se incluyen decisiones en cuanto a su distribución impresa y/o en línea, gratuita o pagada, con o sin políticas de sumisión de artículos, con cesión de derechos o licenciamiento de las obras por los contribuyentes" (Cerda Silva y Lara, 2011, p.5). Estas definiciones deben ser formuladas de acuerdo con los propósitos de la publicación y los intereses, tanto de la institución responsable como de autores y usuarios.

\section{Procedimientos metodológicos}

En la actualidad los problemas de investigación emergen dentro de un contexto organizacional, heterogéneo y circunstancial, cuyo abordaje debe ser transdisciplinar y centrado en sus aspectos sociales. Esta perspectiva es fundamental para la presente investigación, pues el Movimiento de Acceso Abierto tiene como centro el acceso a la información en formato digital a través de la tecnología web e Internet. Sin embargo, la participación en el Movimiento, ya sea como usuarios de la información, como generadores de esta (publicando en revistas de acceso abierto o depositando trabajos en repositorios abiertos) o como intermediarios entre usuarios y autores (comités editoriales, bibliotecas), no depende solamente de la disponibilidad de Tecnologías de la Información (TIC), sino, sobre todo, de otros factores de índole social como la cultura organizacional o disciplinar, los conocimientos y habilidades, el apoyo institucional, entre otras (Sánchez-Tarragó, 2010).

Teniendo en cuenta la perspectiva antes mencionada, para llevar a cabo esta investigación se realizó una triangulación entre métodos cuantitativos y cualitativos. Ambos fueron útiles para la aproximación a las complejas realidades relacionadas con los conocimientos, las percepciones, las actitudes y los comportamientos 
de editores y autores. Las técnicas de recolección y análisis de datos utilizados fueron: (a) revisión bibliográfica sobre el tema de estudio; (b) análisis cuanticualitativo de categorías previamente definidas en las páginas web de los portales de las revistas; (c) encuestas a directores y editores de revistas científicas y a autores con publicación en revistas latinoamericanas; (d) análisis del Registry of Open Access Repository Mandates and Policies (ROARMAP, Registro de Políticas de Acceso Abierto) y (e) grupos focales con editores y autores cubanos (en este artículo no se hará referencia directa a los resultados obtenidos por medio de las dos últimas técnicas).

El análisis cuanti-cualitativo de las páginas web de las revistas se realizó a partir de la codificación de los textos de las páginas Acerca de la revista, Instrucciones o Normas para los autores, Página sobre derecho de autor o Página de aspectos legales en categorías relevantes previamente definidas como revista de acceso abierto; política de acceso abierto; licencia Creative Commons; derecho de autor; copyright, permisos, entre otras. Una vez codificados los textos se realizó la cuantificación de la aparición de estas categorías, así como el análisis cualitativo del contexto y significado de estas en las páginas de las revistas. El análisis se realizó en una muestra de 516 revistas científicas latinoamericanas digitales, seleccionadas aleatoriamente de un universo de 1181 revistas registradas en el Catálogo de Latindex Sistema Regional de Información en Línea para América Latina, el Caribe, España y Portugal y correspondientes a las siete áreas temáticas clasificadas en el sistema. Estos procedimientos permitieron identificar las políticas y permisos asociadas al uso y reúso de los contenidos publicados.

Además, se aplicaron dos cuestionarios autoadministrados, uno para directores de revistas o editores ejecutivos, y otro para autores. Ambos perseguían indagar en las percepciones acerca del modelo de acceso abierto y particularmente, profundizar en las políticas de derecho de autor, los permisos de uso y reúso, y las estrategias de promoción del acceso abierto en las revistas científicas latinoamericanas. Para los directores y editores de revistas se enviaron cuestionarios en español y portugués con invitaciones personalizadas entre junio y octubre del 2013, vía correo electrónico, al
100\% de las direcciones de correo electrónico que aparecían en los 1370 registros del listado que ofrece el catálogo de Latindex.

Para la selección de la muestra de autores se decidió utilizar como criterio que fueran los autores de los artículos con mayor índice-H según los cómputos del sistema de Google Scholar Metrics (GSM). Este índice condensa en una sola magnitud la productividad de los autores con la cantidad de citas recibidas, por lo que garantizaba de manera aproximada que los cuestionarios fueran dirigidos a autores con experiencia en la publicación científica, específicamente en revistas latinoamericanas, y con cierto impacto dentro de su campo. Se seleccionaron las revistas latinoamericanas dentro del ranking que elabora GSM con las 100 revistas con mayor índice-H tanto en idioma español como en idioma portugués, para un total de 123 títulos (Búsqueda realizada el 23 septiembre de 2013). Dentro de cada uno de estos títulos se seleccionaron los seis artículos con más alto índice-H y de allí se extrajeron los nombres y direcciones electrónicas de sus autores. Se enviaron cuestionarios a 662 autores, vía correo electrónico, en septiembre del 2013, en español y portugués.

El enfoque metodológico asumido permitió diagnosticar las condiciones de uso y reúso de los contenidos según la información existente en las páginas web de las revistas, así como confrontar esta información con la perspectiva subjetiva de editores y autores respecto al acceso abierto, y particularmente, respecto a políticas y estrategias editoriales para promover el uso y reúso de la información publicada en acceso abierto. Las tres perspectivas confrontadas (la de los investigadores, como observadores externos, y la de los editores y autores, como actores involucrados directamente en la construcción de estos procesos) permitieron enriquecer y profundizar en el estudio del fenómeno.

\section{Resultados y Discusión}

\section{Análisis de las políticas de derecho de autor de revistas científicas latinoamericanas}

Las 516 revistas estudiadas procedían de 15 países, de ellos, 9 de América del Sur, 1 de América del Norte y 5 de América Central y el Caribe. El 52,7\% eran 
revistas brasileñas, en correspondencia con la proporción de revistas de ese país registradas en el catálogo de Latindex (50,5\%). El 42,4\% de las revistas estaban clasificadas como de Ciencias Sociales, mientras que el resto se distribuyó en Ciencias Médicas (21,3\%), Ciencias Exactas y Naturales (14,9\%), Ciencias de la Ingeniería y Ciencias Agrícolas (ambas con 5,8\%), Artes y Humanidades $(5,4 \%)$ y Multidisciplinarias (4,3\%). Según la tipología de instituciones que utiliza Latindex, el 60,9\% de las revistas estudiadas son editadas por instituciones educativas, fundamentalmente universidades, seguido por el 25,4\% de revistas editadas por asociaciones científicas 0 profesionales.

De las revistas estudiadas, el 99\% ofrecía sus textos completos gratuitamente, ya fuera en el sitio web de la revista, en portales como SciELO y Redalyc, en las plataformas del sistema de gestión electrónica de manuscritos Open Journal System (OJS) u otros. No obstante, únicamente el 19,4\% de las revistas indicaba en su portada que era una revista de acceso abierto.

Un 31,4\% de las revistas no ofrecía ninguna información sobre licencias o acuerdos de derecho de autor. Por otra parte, el $24,0 \%$ de las revistas señalaba sobre las condiciones de uso de sus contenidos: todos los derechos reservados. O sea, utilizaban un acuerdo de derecho de autor completamente restrictivo. Estos resultados coinciden con los hallazgos de Cerda Silva y Lara (2011), que expresaron al respecto: "por omisión o por expresa declaración, un 75,0\% de las publicaciones latinoamericanas en línea analizadas adoptan el modelo legal de licenciamiento de sus contenidos, el que, en síntesis, requiere autorización del titular de los derechos a efectos de hacer uso de la obra" (Cerda Silva \& Lara, 2011, p.16). Aunque en la presente investigación este porcentaje es menor, no deja de ser alarmante.

Un 36,6\% indicaba explícitamente que se ofrecía acceso abierto a los contenidos. De las revistas que indicaban explícitamente ofrecer acceso abierto, el 53,0\% utilizaba la fórmula estándar de la plataforma OJS, y un 32,0\% estaba incluido en la colección SciELO. Del total de las revistas pertenecientes a la colección SciELO - una de las más importantes iniciativas de acceso abierto en la región latino-americana -, alrededor del 37,0\% indicaba en sus páginas Todos los derechos reservados y solicitaba transferencia de derechos de autor. Esto constituye, evidentemente, una contradicción con la propia definición de revista de acceso abierto.

El 35,7\% de las revistas estudiadas mencionaba en su portada la utilización de alguna licencia Creative Commons (CC). El 45,0\% de las revistas que estaban en la colección SciELO indicaban el uso de esta licencia; sin embargo, de las revistas en OJS esta proporción disminuía a 38,0\%. La mayoría de las revistas con licencias CC indicaban el uso de la licencia BY-NC 3.0, aunque muy pocas explicitaban los derechos o permisos específicos que establece esta licencia.

Una de las contradicciones más importantes detectadas fue la utilización mezclada de licencias Creative Commons junto con la declaración de Todos los derechos reservados en páginas de muchas revistas, incluyendo aquellas hospedadas en la colección SciELO. Este aspecto también había sido identificado por Cerda Silva y Lara (2011), alertando sobre la incertidumbre en cuanto a las condiciones bajo las cuales está disponible la publicación.

A partir de la información legal disponible en las revistas estudiadas, los investigadores evaluaron si la misma aparecía de manera explícita, poco explícita o nada explícita. La información se consideró poco explícita en más de la mitad de las revistas (51,0\%) pues, o bien solamente declaraban Todos los derechos reservados, o bien utilizaban la fórmula estándar disponible en la plataforma Open Journal System que declara"esta revista provee acceso libre inmediato a su contenido bajo el principio de que hacer disponible gratuitamente investigación al público, apoya a un mayor intercambio de conocimiento global", o indicaban usar una licencia Creative Commons, sin plantear, en cualquiera de estos casos, los derechos o permisos específicos para autores y lectores. En el 33,7\% de las revistas, la información sobre los permisos se consideró nada explícita al no aparecer ninguna información sobre derecho de autor, declaración de licencia Creative Commons u otra ni información adicional sobre este aspecto.

\section{Perspectiva de editores de revistas científicas latinoamericanas}

Los 400 cuestionarios recibidos ( 33\% de respuestas) procedían de dieciséis países (Argentina [11,0\%], Brasil [33,8\%], Colombia [3,3\%], Costa Rica [2,8\%], 
Cuba [6,3\%], Chile [10,3\%], Ecuador [0,3\%], El Salvador [0,3\%], México [21,8\%], Nicaragua [0,3\%], Panamá [0,3\%], Paraguay [0,5\%], Perú [3,0\%], Puerto Rico [0,5\%], Uruguay [1,5\%] y Venezuela [4,5\%]). Se obtuvieron respuestas de editores de todas las áreas disciplinares, aunque las mejor representadas fueron las Ciencias Sociales (44,5\%), las Ciencias Exactas y Naturales (16,5\%) y las Ciencias Médicas (16,0\%). Alrededor del 80\% de los cuestionarios recibidos fue rellenado por las personas que ocupaban los cargos de mayor responsabilidad en la revista (director o editor ejecutivo). El 57,0\% de ellos tenía experiencia relativamente corta en esa responsabilidad (1 a 5 años).

El 98,5\% de los encuestados consideró que su revista se distribuía gratuitamente en Internet, y un porcentaje ligeramente menor $(95,7 \%)$ contestó que su revista era de acceso abierto. Por tanto, no todas las revistas digitales y gratuitas son consideradas por su comité editorial como revistas de acceso abierto.

La mayoría de los encuestados señaló que sus revistas basaban los acuerdos de derecho de autor en la transferencia de los derechos a la revista (58\%), mientras que un 33\% indicó utilizar alguna licencia Creative Commons. Hubo 19 encuestados que dijeron utilizar ambos tipos de acuerdos, entre los cuales algunos especificaron que utilizaban la transferencia de derechos para la versión impresa de la revista y la licencia CC para la versión electrónica.

Entre los resultados contradictorios obtenidos en nuestro estudio está que, del total de editores que indicaron utilizar licencias Creative Commons, menos de la tercera parte señaló que la revista concedía todos los posibles permisos mencionados en el cuestionario, aún cuando estos están incluidos en cualquiera de las variantes de una licencia CC. Por tanto, es posible que la adopción de licencias CC por parte de los comités editoriales de las revistas no esté acompañada por un conocimiento real de los permisos que se están concediendo a autores y lectores.

En el cuestionario también se enunció un grupo de estrategias o iniciativas que podrían utilizar los comités editoriales o la institución responsable de la revista para promover el modelo de acceso abierto entre los autores potenciales, y se solicitó a los editores señalar aquellas que se utilizaban. La respuesta seleccionada por la mayoría de los encuestados fue "Se incluyó a la revista en directorios de acceso abierto" (71\%), seguida por "Establecimos una política de acceso abierto" (61\%). Estas respuestas denotan escasez de iniciativas si tenemos en cuenta que la lista de contenía ocho opciones.

Se indagó sobre las barreras percibidas por los editores para desarrollar el modelo de acceso abierto en las revistas latinoamericanas. Esta pregunta abierta acumuló 384 opiniones que fueron agrupadas por los investigadores en categorías; entre ellas, la que acumuló mayor cantidad de criterios fue Financiamiento, seguida por Familiarización con el modelo, Patrones culturales, Habilidades y competencias, Calidad de las revistas y Sistema de recompensa científica. Dos comentarios verbales de los editores ejemplifican las barreras percibidas y que están relacionadas con la tensión existente entre el modelo tradicional de revistas por suscripción y el modelo de acceso abierto, así como la poca familiarización de los editores con este último.

\begin{abstract}
[...] La barrera tiene que ver con la existencia de una institucionalidad que favorece revistas que utilizan copyright, que generalmente son las de mayor prestigio, por sobre aquellas que utilizan otro de tipo de licencias [...] Por otra parte, existe mucho desconocimiento en el medio académico respecto a los elementos positivos del modelo de acceso abierto, y no existe ningún tipo de incentivo por preferir ese tipo de modelo por sobre uno basado en el copyright. Por lo tanto, muchas veces, incluidos nosotros como revista académica, descuidamos el tema de las licencias $y$, en nuestro caso, simplemente no hemos explicitado con qué licencia trabajamos [...].

[...] No hay una vinculación entre la política de la universidad, la casa editora y el modelo de acceso abierto. Aunque la promoción y subvención de la edición de las revistas está enunciado en un reglamento, no se especifica ningún modelo editorial al respecto [...].
\end{abstract}

\section{Perspectiva de los autores}

De los 101 autores que respondieron el cuestionario ( $\sim 15 \%$ de respuestas), 47,5\% residían en Brasil; 13,9\% en México y otros porcentajes menores en Colombia, Argentina, Cuba, España, Chile, Perú, Francia y Alemania. Los resultados obtenidos indican que la mayoría, con una trayectoria profesional y científica importante, conocen revistas de acceso abierto en su campo de investigación 
(93,0\%) y han tenido experiencia de publicación en estas (89,0\%), incluyendo revistas latinoamericanas $(69,0 \%)$. Estos resultados coinciden con estudios recientes realizados con investigadores argentinos (Bongiovani et al., 2012). Del total de artículos que los autores encuestados publicaron en 2012, alrededor del 53,0\% fueron publicados en revistas de acceso abierto. No obstante, algunos de los autores manifestaron no estar seguros de si la revista donde habían publicado era de acceso abierto o no. Estos números implican que, aún cuando todos los autores encuestados publican en revistas latinoamericanas (por esta razón fueron seleccionados en la muestra), alrededor de un 30,0\% de ellos no las identifica como revistas de acceso abierto o no está seguro de que lo sean; esto es otra señal de la necesidad de mayor información y políticas editoriales más claras al respecto.

El 56\% de los autores encuestados señaló haber examinado los derechos de autor en el contrato entregado por la revista y haberlo firmado sin modificaciones; sin embargo, un 20\% de ellos firmó el contrato sin haberlo examinado. Este aparente desinterés en los aspectos legales podría estar contribuyendo a los resultados que se discuten con más detalle en la sección siguiente.
Por otra parte, el 45,0\% de los encuestados manifestó no tener información sobre estrategias institucionales para promover el acceso abierto, no obstante, el establecimiento de revistas de acceso abierto $(35,5 \%)$ y la implantación de repositorios institucionales (30,0\%) estuvieron entre las escogidas por el resto. Este limitado conocimiento sobre estrategias podría constituir señal de cierto "silencio" o inercia con respecto a estos temas en parte de las instituciones de la región (al menos a nivel local) o, peor aún, que los esfuerzos institucionales a favor del acceso abierto no tienen suficiente visibilidad e influencia en los autores.

\section{Permisos concedidos por las revistas según percepciones de autores y editores}

Un aspecto que interesaba explorar era la percepción de editores y autores respecto a los permisos concedidos por las revistas. Para ello, se pidió a los editores que seleccionaran de una lista de posibles permisos, aquellos que su revista concedía a los autores. Esa misma lista se presentó a los autores para que valoraran cuáles eran los permisos más importantes y cuáles de ellos habían retenido en los artículos publicados en el 2012 (Tabla 1).

Tabla 1. Percepciones de autores y editores acerca de permisos para usar los artículos publicados.

\begin{tabular}{|c|c|c|c|c|}
\hline Permisos & $\begin{array}{l}\text { Editores que } \\
\text { indicaron que sus } \\
\text { revistas lo conceden }\end{array}$ & $\begin{array}{l}\text { Autores que consideran que } \\
\text { es muy importante o } \\
\text { importante retenerlo }\end{array}$ & $\begin{array}{l}\text { Autores que } \\
\text { consideran que } \\
\text { lo retienen }\end{array}$ & $\begin{array}{c}\text { Autores } \\
\text { inseguros de } \\
\text { haberlo retenido }\end{array}$ \\
\hline $\begin{array}{l}\text { Enviar una copia del artículo por correo electrónico } \\
\text { a otros colegas. }\end{array}$ & 63 & 79 & 63 & 26 \\
\hline $\begin{array}{l}\text { Permiso para que los demás miembros de su } \\
\text { institución utilicen copias del artículo con propósitos } \\
\text { de enseñanza. }\end{array}$ & 66 & 74 & 58 & 30 \\
\hline $\begin{array}{l}\text { Depositar el artículo publicado (una versión creada } \\
\text { por el autor) en un repositorio de acceso aberto. }\end{array}$ & 49 & 71 & 38 & 38 \\
\hline $\begin{array}{l}\text { Reutilizar parte o la totalidad del artículo en sus } \\
\text { artículos o libros futuros. }\end{array}$ & 44 & 67 & 43 & 38 \\
\hline $\begin{array}{l}\text { Distribuir fotocopias del artículo publicado entre } \\
\text { sus estudiantes. }\end{array}$ & 60 & 66 & 61 & 24 \\
\hline $\begin{array}{l}\text { Colocar el artículo publicado (la versión del editor) } \\
\text { en su sitio Web personal. }\end{array}$ & 56 & 50 & 35 & 39 \\
\hline $\begin{array}{l}\text { Colocar el artículo (una versión creada por el autor) } \\
\text { en su sitio Web personal. }\end{array}$ & 44 & 44 & 33 & 39 \\
\hline
\end{tabular}

Fuente: Elaboración propia (2013)

Nota: Porcentajes redondeados. 
En general, se aprecia la falta de correspondencia entre las expectativas de los autores respecto a los usos posteriores sobre sus trabajos publicados y su percepción acerca de los permisos que les fueron concedidos. Por ejemplo, resulta interesante comparar los permisos que concedieron los editores y la importancia que otorgan los autores a la retención de estos. Véase que las divergencias son inferiores a 10\% en los permisos 2, 5, 6 y 7; y mayores - más de 20\% - en los permisos 3 y 4 . En general las aspiraciones de los autores son mayores o iguales a las concesiones de los editores, excepto en el permiso 6. Diferente sucede respecto a los derechos retenidos por los autores, donde el grado de desencuentro respecto a los editores varía de $1 \%$ o menos (permisos 1 , 4,5 ), pasando por 10\% (permisos 2, 3, 7) hasta alcanzar el $15 \%$ en el permiso 6.

Se observa también gran inseguridad en los autores respecto a los permisos retenidos, que predomina en más de un tercio de los encuestados cuando se trata de colocar el artículo en su sitio web personal, reutilizar parte o la totalidad del artículo en sus artículos o libros futuros y depositar el artículo publicado (una versión creada por el autor) en un repositorio de acceso abierto. Sin embargo, resulta interesante el contraste con las opiniones de los editores. Aunque en casi ningún caso los permisos concedidos se acercan a las expectativas de los autores, en la mayoría de ellos los permisos que se dicen conceder superan las percepciones de los autores acerca de cuáles han sido retenidos.

La interpretación de estos resultados debe tener en cuenta que, tal como algunos estudios han apuntado (Swan, 2008; Fry et al., 2009; Dallmeier-Tiessen et al., 2011), muchos autores no están familiarizados ni prestan suficiente atención a los aspectos relacionados con el derecho de autor y los permisos que conceden las revistas. De hecho, el presente estudio encontró que al menos un 20\% de los autores encuestados había firmado el contrato de derecho de autor sin revisarlo. No obstante, tal como ocurre con el estudio similar de Morris (2009), el contraste entre ambas perspectivas (autores y editores) tiene valor como indicador de una situación de conflicto.

Como se observa en la Tabla 2, las tres cuartas partes de los editores encuestados considera que la información sobre los permisos está suficientemente explícita en las revistas (74\%), lo quecontrasta notablemente con el análisis realizado por los investigadores (15\%). No obstante, a la luz de las divergencias entre las percepciones de los editores y los autores respecto a los permisos concedidos y retenidos, es posible afirmar que sí existe un problema importante con la claridad en que son expuestas estas informaciones en las revistas o con las habilidades de los autores para comprenderlas, ya que, interrogados estos últimos sobre este aspecto, el 57\% consideró difícil o muy difícil comprender los aspectos legales relacionados con el derecho de autor y las licencias para publicar en las revistas científicas.

Las discrepancias o confusiones entre lo que establecen o asumen los editores con respecto al derecho de autor y las expectativas y necesidades de los autores han sido discutidos por otros autores como Swan (2008) y Morris (2009). Al igual que estas autoras, consideramos que entre los factores que pueden influir en estas contradicciones está que los editores no han comunicado con efectividad sus políticas de derecho de autor o su alineación coherente con el modelo de acceso abierto. Tal como sucedía con las revistas españolas estudiadas por Melero et al. (2009), aún se requiere un

Tabla 2. Percepciones de los actores del acceso abierto acerca de las informaciones sobre permisos para usar los artículos publicados que se ofrecen en las revistas.

\begin{tabular}{|c|c|c|c|}
\hline $\begin{array}{l}\text { Los permisos que se concedena autores y } \\
\text { lectores están }\end{array}$ & Percepción de losEditores & $\begin{array}{l}\text { Percepción de los Autores } \\
\end{array}$ & Análisis de los Investigadores \\
\hline Nada explícitos & 6 & 7 & 34 \\
\hline Poco explícitos & 19 & 37 & 51 \\
\hline Explícitos & 74 & 56 & 15 \\
\hline
\end{tabular}

Fuente: Elaboración propia (2013).

Nota: Porcentajes redondeados. 
esfuerzo mayor por parte los directores y editores de las revistas latinoamericanas para que sus políticas editoriales, incluyendo las políticas de derecho de autor y las referidas al acceso abierto, expresen inequívocamente y con claridad, de manera fácilmente accesible, el modelo de financiamiento y licenciamiento que emplea la revista. Esto implica que los editores necesitan mejor preparación para definir sus políticas y ofrecer información apropiada sobre ellas. Asimismo, los autores requieren más información y capacitación sobre estos temas y más empoderamiento para asumir conscientemente sus derechos como autor.

Por otra parte, la aparente contradicción entre la percepción generalizada de los editores de que sus revistas son de acceso abierto, el hecho real de que se ofrecen gratuitamente, y a la vez, el desconocimiento y la confusión sobre las políticas de derecho de autor, en opinión de los autores del presente estudio, pudiera sugerir que las licencias restrictivas 'Todos los derechos reservados' no forman parte de la cultura de las publicaciones científicas en la región de América Latina. Con relación a esto, uno de los aspectos a tener en cuenta es la forma particular en que evolucionó la comunicación científica en América Latina, que si bien pasó por etapas de desarrollo similares a las de Europa y los Estados Unidos, o sea primeramente a través de sociedades científicas y luego, de universidades e instituciones académicas (Cetto \& Alonso-Gamboa, 1998), no llegó a concretarse a través de industrias editoriales privadas (Quartesan et al., 2007). Nuestra investigación encontró que un $60,9 \%$ de las revistas son editadas por instituciones educativas, fundamentalmente universidades, seguido por el 25,4\% de revistas editadas por asociaciones científicas o profesionales, y el resto de los financistas, en menores proporciones. Lo anterior muestra una diferencia palpable con la tendencia mundial, donde más del 60,0\% de las revistas científicas son publicadas por editoriales comerciales (Crow, 2006). Por otra parte, es imprescindible tener en cuenta que el derecho de autor surgió específicamente en Europa, bajo la economía de mercado capitalista, por lo que este régimen de protección y sus licencias están permeados de la visión europea hacia la creación, en la cual la propiedad privada y el mercado desempeñan un papel fundamental (Story et al., 2006). Según el criterio y la experiencia de los autores de este trabajo, en el caso de las publicaciones científicas, la visión latinoamericana del derecho de autor difiere de esa visión europea de 'Todos los derechos reservados', lo que ha propiciado que el acceso gratuito a las publicaciones científicas haya existido en esta región incluso antes del surgimiento formal del Movimiento de Acceso Abierto (Sánchez-Tarragó et al., 2012a, 2012b; Trzesniak, 2012).

No obstante, a nuestro juicio, la mayor parte de los autores y de los propios editores solamente tienen el concepto restringido de acceso abierto que considera que basta con hacer disponible los artículos en formato electrónico. Por tanto, consideramos que la adopción consciente y definitiva de políticas y modelos de licenciamiento por parte de las revistas latinoamericanas que garanticen la mayor flexibilidad de permisos y derechos a los autores y lectores sobre los contenidos publicados, es la manera más coherente de ordenar y darle continuidad al modelo de acceso abierto que se ha venido desarrollando en la región de América Latina de manera espontánea en sus inicios y, desde hace unos años, a través de numerosas iniciativas de gran repercusión para la visibilidad y accesibilidad de la producción científica latinoamericana.

A la luz del análisis de las bases de poder realizado por Reinsfelder (2012) y del propio marco teórico que apoya esta investigación, consideramos que el éxito del acceso abierto en las revistas latinoamericanas depende de un conjunto de estrategias dirigidas a fomentar acciones de participación conjunta entre los actores que conforman el sistema de comunicación científica y que deben abarcar las dimensiones políticas, de calidad y de gestión editorial entre otras. Por ejemplo, las instituciones científicas y académicas deberían implementar políticas y reglamentos que refuercen el acceso abierto; desarrollar nuevos canales y servicios para la diseminación de los resultados en acceso abierto; adherirse públicamente a declaraciones a su favor y establecer líneas o comités de investigación sobre estas nuevas prácticas. Los comités editoriales y las instituciones (incluyendo sus bibliotecas) deberían impartir conferencias sobre estos temas, premiar o estimular a los autores que utilizan esta vía de diseminación, así como establecer servicios de asesoría sobre derecho de autor. 
Las instituciones y agencias que financian investigaciones en nuestra región tienen un papel muy importante en la aspiración de maximizar el acceso y uso de los resultados científicos en Latinoamérica, por lo que deberían establecer políticas que promuevan y estimulen a los autores a publicar en revistas latinoamericanas de acceso abierto: cubrir los costos de publicación de los autores e instituciones que deseen publicar en las revistas que apliquen tarifas por el procesamiento de los artículos y su publicación en acceso abierto; sustituir el criterio utilizado actualmente por gran parte de las instituciones académicas y de investigación, relativo a la obligatoriedad de publicar en revistas de corriente principal y de alto factor de impacto, por el criterio del mérito intrínseco del resultado de investigación y la utilización de índices de evaluación alternativos. A todas luces, cualquier estrategia a favor del acceso abierto debería contemplar en primer lugar la elevación de la calidad de los procesos editoriales. La baja calidad de estos debilita la utilización y promoción de las revistas latinoamericanas como vehículo de diseminación de los resultados científicos. Este planteamiento destaca la necesidad de, por una parte, reforzar la formación de competencias en los editores y equipos editoriales y, por otra, la implantación de normas y políticas editoriales que garanticen un marco de conducta explícito y estándar (Trzesniak et al., 2012).

Finalmente, si queremos que las revistas latinoamericanas asuman el modelo de acceso abierto sin ambigüedades, los editores y comités editoriales deberían hacer un esfuerzo intensivo para alinear explícita y definitivamente sus políticas editoriales (incluyendo aquellas que definen derecho de autor $y$ condiciones de uso) a un modelo de licenciamiento flexible que garantice el acceso a los contenidos sin barreras económicas, tecnológicas y legales, dentro de un marco ético y de atribución adecuada de la autoría. Estas licencias deben aparecer en los sitios web de las revistas y brindar información explícita sobre los permisos de autores y lectores. Un papel importante en este sentido lo desempeñan los sistemas regionales de información, indexación y publicación como Latindex, Redalyc, SciELO, cuyos controles de calidad rigurosos deben contemplar la estandarización de la información que debe registrarse en las plataformas de acceso de las revistas. En esta misma línea, las instituciones y los propios comités editoriales deben realizar mayores esfuerzos para la formación de los autores con respecto a modelos de licenciamiento que garantizan mayor accesibilidad y visibilidad a sus artículos. Debería incentivarse el uso de instrumentos tales como SPARC Autor Addendum <http://www.sparc.arl.org/resources/authors/ addendum>, que guían a los autores en la modificación de acuerdos de derecho de autor con los editores.

\section{Conclusión}

En los últimos años, el modelo de acceso abierto ha tenido un impacto importante en el sistema de comunicación científica, lo que se evidencia en el volumen de literatura publicada sobre el tema, el crecimiento de las publicaciones y repositorios de acceso abierto, la diversificación de los modelos de financiamiento, la flexibilización de las licencias de derecho de autor, la implementación de políticas de acceso abierto por parte de importantes agencias financistas gubernamentales y el aumento de la familiarización y de las prácticas de comunicación científica, utilizando este modelo por parte de los investigadores y sus instituciones. No obstante, este avance no es igual en todas las regiones, países, ni disciplinas académicas, lo que se corresponde también con las diferencias en las culturas epistémicas y los desarrollos políticos y de las organizaciones en cada contexto específico. Persisten también prejuicios culturalesy limitaciones relacionadas con el financiamiento, el marco legal y la gestión editorial, entre muchos otros.

La presente investigación permitió constatar que, aunque la mayoría de las revistas científicas latinoamericanas en línea son consideradas por sus editores como revistas de acceso abierto, una parte importante de ellas mantiene un modelo de licenciamiento de sus contenidos incompatible o contradictorio con la definición de publicación de acceso abierto, ya que restringe el uso de sus contenidos o son poco explícitos acerca de los derechos que autores y lectores tienen sobre los artículos publicados. Son pocas también las estrategias editoriales encaminadas a promover y darle visibilidad al modelo de acceso abierto, lo que está relacionado con el propio desconocimiento de los 
comités editoriales y las instituciones responsables. Los cambios culturales que se requieren para transformar actitudes y prácticas vienen de la mano con la información y la preparación de editores, autores y dirigentes administrativos de las instituciones que patrocinan estas revistas.

A nuestro juicio, una amenaza importante estriba en el desconocimiento de autores y editores de lo que representa el concepto de acceso abierto. Cuando asumen que se trata solamente de ofrecer el contenido en un sitio web, no visualizan el contexto completo descrito en la definición de la BOAl que se ofreció en la Introducción de este trabajo y no tienen tampoco la noción de interoperabilidad y de la importancia de ofrecer metadatos de calidad. Internet abrió la perspectiva de que la responsabilidad por la comunicación científica volviera a ser de los científicos, y posibilitó la retirada de las editoras comerciales, pero esto implica compromisos y deberes que los actores no están cumpliendo adecuadamente por desconocimiento, desinterés u otras razones. Esta situación de inercia puede llevar al fracaso del acceso abierto y al retorno de una dependencia perversa a las editoriales comerciales.

Finalmente, parece particularmente interesante profundizar en investigaciones futuras en las condicionantes socioeconómicas y epistémicas que propiciaron el surgimiento del acceso abierto en América Latina y en su sostenibilidad. ¿Están conscientes los autores, editores, responsables de instituciones académicas, decisores, qué hay detrás de esta tradición y lo que esto implica para la ciencia latinoamericana? ¿Cómo salvaguardar esta tradición en medio de un mundo cada vez más globalizado y mercantilizado, donde también el conocimiento y la educación se han convertido en bienes comercializables, y donde la competencia ha alcanzado a universidades y centros de investigación que se resisten en compartir información y conocimiento cuando en ellos hay un filón comercializable? En nuestra opinión, además de estrategias de tipo operativo (como incorporación a directorios de acceso abierto o modificación de licencias de derecho de autor), habría que ir en pos de otras encaminadas a hacer consciente, honorable y valioso el acto de compartir y devolverlo al centro de la ética y la actuación de los investigadores de la región, todo ello en un marco de apoyo político e institucional.

\section{Agradecimiento}

A los autores, editores y directores de las revistas analizadas en este estudio.

Esta investigación fue apoyada por el proyecto Quality in the Open Scholarly Communication of Latin America: IDRC Grant reference number: 106660-001 y la Facultad Latinoamericana de Ciencias Sociales (FLACSO), Brasil.

\section{Colaboraciones}

N. SÁNCHEZ-TARRAGÓ, J.C. FERNÁNDEZ-MOLINA e A. C. RIVERO contribuyeron em la concepción y diseño del estudio, análisis de datos y redacción final. D. DEROY contribuyó en la recogida de datos y su análisis. P. TRZESNIAK contribuyó con el análisis de datos y redacción final. R.N.M. SANTOS contribuyó con la redacción y revisión final. Todos los autores revisaron y aprobaron la versión final.

\section{Referencias}

Babini, D. Acceso abierto a la producción científica de América Latina y el Caribe: identificación de principales instituciones para estrategias de integración regional. Revista CTS, v.17, n.6, p.31-56, 2011. Disponible en: <http://revistacts.net/files/ Volumen\%206\%20-\%20N\%C3\%BAmero\%2017/Babini.pdf>. Acceso en: 20 enero 2015.

Björk, B.C. Open access to scientific publications: An analysis of the barriers to change. Information Research, n.9, 2004. Available from: <http://informationr.net/ir/92/paper170. html>. Cited: May 27, 2015.

Bongiovani, P. Gómez, N-D.; Miguel, S. Opiniones y hábitos de publicación en acceso abierto de los investigadores argentinos: un estudio basado en los datos de la encuesta
SOAP. Revista Española de Documentación Científica, v.35, n.3, p.453-467, 2012.

Budapest Open Access Initiative. Read the Budapest Open Access Initiative. 2002. Available from: <http://www.soros.org/ openaccess/read.shtml>. Cited: Jan. 20, 2015.

Budapest Open Access Initiative. Ten years on from the Budapest Open Access Initiative: Setting the default to open. 2012. Available from: <http://www.budapestopenaccessinitiative. org/boai-10-recommendations>. Cited: May 27, 2015.

Carroll, M.W. Creative Commons and the openness of open access. The New England Journal of Medicine, n.368, p.789-791, 2013. 
Cerda Silva, A.; Lara, J.C. Políticas editoriales de publicaciones académicas en línea en Latinoamérica. Informe final. Santiago de Chile: ONG Derechos digitales, 2011. Disponible en: <http:// www.revistasabiertas.com/wp-content/uploads/InformePoliticas_Editoriales_de_Publicacio nes.pdf>. Acceso en: 20 enero 2015.

Cetto, A.M.; Alonso-Gamboa, O. Scientific periodicals in Latin America and the Caribbean: A global perspective. Interciencia, v.23, n.2, 1998. Available from: <http://www.researchgate.net/ publication/237253705_scientific_periodicals_in_latin_ america_and_the_caribbean_a_global_perspective $>$. Cited: Jan. 20, 2015

Crow, R. Publishing cooperatives: An alternative for non-profit publishers. First Monday, v.11, n.9, 2006. Available from: <http:// firstmonday.org/ojs/index.php/fm/article/view/1396/1314>. Cited: Jan. 20, 2015.

Dallmeier-Tiessen, S. et al. Open access journals: What publishers offer, what researchers want. Information Services \& Use, v.31, p.85-91, 2011

Directory of Open Access Journals. Copyright and licensing incompatibility. Part 1, 2015. Available from: <https:// doajournals.wordpress.com/2015/05/19/copyright-andlicensing-incompatibility-part-1/>. Cited: Jun. 6, 2015

Fry, J. et al. PEER Behavioural Research: Authors and users visàvis journals and repositories, 2009. Available from: <http:// wWw.stm-assoc.org/2010_01_20_Final_revision_ behavioural_baseline_report.pdf $>$. Cited: Jun. 6, 2015.

Gómez, N-D. et al. Los nuevos canales de comunicación de la ciencia y la respuesta de los científicos chilenos. In: Seminario Nuevas Tendencias en Información y sus Implicancias en el Desarrollo Profesional Bibliotecario, 2008, Anales electrónicos... Santiago: e-Lis, 2008. Disponible en: <http://eprints.rclis.org/ 14635/>. Acceso en: 6 jun. 2015.

Gómez, N-D. et al. Open access indicators and information society: The Latin American case. OCLC Systems \& Services, v.25, n.2, p.8292, 2009

Harnad, S. Opening access by overcoming Zeno's paralysis. In: Jacobs, N. (Ed). Open access: Key strategic, technical and economic aspects. Oxford: Chandos Publishing, 2006. Available from: <http://eprints.ecs.soton.ac.uk/12094/>. Cited: Jun. 6, 2015.

Melero, R. et al. DULCINEA: Copyright policies and type of access to Spanish scientific journals. 2009. Available from: <http://elpub.scix.net/data/works/att/89_elpub2009. content.pdf>. Cited: Jun. 6, 2015.

Miguel, S.; Gomez, N-D.; Bongiovani, P. Prospect for development of open access in Argentina. The Journal of Academic Librarianship, v.39, n.1, p.12, 2013.

Morris, S. Journal authors'rights: Perception and reality. London: Publishing Research Consortium, 2009. Available from: <http:// publishingresearchconsortium.com/index.php/110-prcprojects/summary-papers/journal-authors-rights-summarypaper/140-journal-authors-rights-percept ion-and-reality>. Cited: Jun. 6, 2015

Pinch, T.J.; Bijker, W.E. The social construction of facts and artifacts: Or how the sociology of science and the sociology of technology might benefit each other. In: Bijker, W.E. et al. (Ed.).
The social construction oftechnological system: New directions in the sociology and history of technology. Cambridge: MIT Press, 1987. p.17-50.

Quartesan, A. et al. Cultural Industries in Latin America and the Caribbean: Challenges and opportunities. 2007. Available from: $<$ http://publications.iadb.org/handle/11319/1143?localeattribute=en $>$. Cited: Jan. 20, 2015.

Reinsfelder, T.L. Open access publishing practices in a complex environment: Conditions, barriers, and bases of power. Journal of Librarianship and Scholarly Communication. v.1, n.1, 2012. Available from: <http://jlsc-pub.org/cgi/viewcontent.cgi? article=1029\&context=jlsc $>$. Cited: Jan. 20, 2015.

Sánchez-Tarragó, N. Política de acceso abierto a la producción científica del Sistema Nacional de Salud, 2010. Tesis (Doctorado en Ciencias de la Información) - Universidad de Granada, España. Disponible en: <http://hera.ugr.es/tesisugr/195 63231.pdf>. Acceso en: 27 mayo 2015.

Sánchez-Tarragó, N.; Fernández-Molina, J.C. Open access journals: Knowledge and attitudes among Cuban health researchers. MEDICC Review, v.10, n.1, p.18-21, 2008.

Sánchez-Tarragó, N.; Fernández-Molina, J.C. The open access movement and Cuban health research work: An author survey. Health Information and Libraries Journal, v.27, n.1, p.66-74, 2010.

Sánchez-Tarragó, N. et al. Reflexiones en torno al acceso abierto a la información en el contexto cubano: el caso del sector salud. Informação \& Sociedade: Estudos, v.22, n.2, p.51-59, 2012a. Disponible en: <http://www.ies.ufpb.br/ojs/index.php/ ies/article/view/10499>. Acceso en: 20 jan. 2015.

Sánchez-Tarragó, N. et al. An open access policy for the scientific output of Cuba's National Health System. Libri, v.62, n.3, p.211-221, 2012b.

Sánchez-Tarragó, N. et al. Políticas institucionales y editoriales que favorecen el acceso abierto a la información (Informe final). 2013. Disponible en: <http://dx.doi.org/10.6084/m9.figshare. 1038870>. Acceso en: 12 enero 2016.

Story, A. et al. The Copy/South dossier: Issues in the economics, politics and ideology of copyright in the Global South. 2006. Available from: <http://kar.kent.ac.uk/6/1/CSdossier.pdf>. Cited: Jan. 20, 2015.

Suber, P. Open access. Massachussett: MIT Press, 2012.

Swan, A. Key concerns within the scholarly communication process: Report to the JISC Scholarly Communications Group. Truro: Key Perspectives Ltd, 2008. Available from: <http:// www.keyperspectives.co.uk/openaccessarchive/reports. html>. Cited: May 202015.

Swan, A.; Brown, S. Open access selfarchiving: An author study. Truro: Key Perspectives, 2005. Available from: <http:// eprints.ecs.soton.ac.uk/10999/1/jisc2.pdf>. Cited: May 20 2015.

Trzesniak, P. A questão do livre acesso aos artigos publicados em periódicos científicos. Em aberto, v.25, n.87, p.77-112, 2012. Disponível em: <http://emaberto.inep.gov.br/index. php/emaberto/article/view/2620/1802>. Acesso em: 20 jan. 2015. 
Trzesniak, P. et al. Qualidade de conteúdo: o grande desafio para os editores científicos. Revista Colombiana de Psicologia, v.21, n.1, p. 57-75, 2012. Disponível em: <http://www.revistas. unal.edu.co/index.php/psicologia/article/view/31032/31 098> Acesso em: 27 mar. 2016

Villarroya, A. et al. Modelos de negocio de las editoriales de revistas científicas: implicaciones para el acceso abierto. El profesional de la Información, v.21, n.2, 2012, p.129-135.
Ware, M.; Mabe, M. The STM report: An overview of scientific and scholarly journal publishing: International Association of Scientific, Technical and Medical Publishers, 2012. Available from: <http://www.stm-assoc.org/2012_12_11_STM_ Report_2012.pdf>. Cited: Jan. 20, 2015.

Xia, J. A longitudinal study of scholars attitudes and behaviors toward open-access journal publishing. Journal of the American Society for Information Science and Technology, v.61, n.3, p.615-624, 2010. 